\title{
CORNEAL OEDEMA AFTER CATARACT SURGERY- AN OVERVIEW
}

\author{
Abhishek Ranjan ${ }^{1}$
}

${ }^{1}$ Senior Resident, Department of Ophthalmology, Patna Medical College Hospital, Patna, Bihar, India.

\section{ABSTRACT}

\section{BACKGROUND}

Phacoemulsification is the most commonly performed cataract surgery now a days. Most of the patients are able to achieve excellent visual outcome due to availability of advanced equipment. Corneal oedema after phacoemulsification in the immediate postoperative period often leads to patient dissatisfaction and worsening of outcome. Thorough pre-operative workup is important to find out any risk factor which is associated with high chances of postoperative corneal oedema. Aetiological factors of early onset corneal oedema must be identified and dealt accordingly. In the early stage, medical management or minor surgical interventions are sufficient to resolve the condition. Delayed onset corneal oedema often requires endothelial keratoplasty. This review highlights the etiopathogenesis, risk factors, investigative modalities and management of corneal oedema in the acute and late phase.

\section{KEY WORDS}

Corneal Oedema, Descemet Membrane Detachment, Phacoemulsification, Toxic Anterior Segment Syndrome.

HOW TO CITE THIS ARTICLE: Ranjan A. Corneal oedema after cataract surgery- an overview. J. Evolution Med. Dent. Sci. 2019;8(07):463-466, DOI: 10.14260/jemds/2019/102

\section{BACKGROUND}

The art of phacoemulsification surgery has evolved over a period of time and perfecting itself in every aspect. Corneal oedema following phacoemulsification is a postoperative complication that may occur in some cases. In the age where cataract surgery is deemed to have vision par perfection, the slightest error can leave a patient in misery. Even immediate blurry vision, however temporary, is accountable for. Postoperative corneal oedema, hence, can plague even the most proficient of surgeons.

\section{Etiopathogenesis}

The light transmissibility of human cornea has been accredited to the lattice arrangement of collagen fibrils, with consequent minimization of light scattering and destructive interference at the stromal level in combination with corneal crystallins; and the relative state of dehydration maintained at the endothelial level by an array of molecular pumps, chemical modulators and cell junctional properties. ${ }^{1}$

Among the several myriad complications that might occur during a cataract surgery, corneal oedema is frequently encountered on. The post-phacoemulsification corneal oedema may occur due to endothelial pump failure following surgery, which may be due to mechanical injury, chemical injury, subsequent infection/inflammation, or concurrent/pre-existing endothelial compromise. ${ }^{2}$

\section{Risk Factors}

The risk factors [Table 1] for postoperative phacoemulsification corneal oedema include the following:

'Financial or Other Competing Interest': None.

Submission 18-01-2019, Peer Review 06-02-2019,

Acceptance 09-02-2019, Published 18-02-2019.

Corresponding Author:

Dr. Abhishek Ranjan,

Senior Resident

Department of Ophthalmology,

Patna Medical College Hospital,

Patna-80004,

Bihar, India.

E-mail: docabhiran@gmail.com

DOI: $10.14260 /$ jemds $/ 2019 / 102$

\begin{tabular}{||lll}
\hline \hline Preoperative & Intra-operative & Postoperative \\
\hline Endothelial dystrophy & Instrument & Vitreous in anterior \\
CHED & trauma & chamber \\
FECD, ICE syndrome & Irrigating & IOL - endothelial touch \\
PPMD & solutions toxicity & Toxic anterior \\
Pseudoexfoliation & Excessive use & segment syndrome \\
Hard cataract & of phaco power & Raised intraocular \\
Chronic uveitis & Intracameral & pressure \\
Chronic angle-closure & drugs toxicity & Chronic inflammation \\
glaucoma & DMD & Brown McLean \\
Trauma to corneal & IOL-related & syndrome \\
endothelium & factors & Wound leak/shallow \\
& & chamber/hypotony \\
\hline
\end{tabular}

CHED: Congenital hereditary endothelial dystrophy, FECD: Fuchs endothelial corneal dystrophy, ICE: Iridocorneal endothelial, PPMD: Posterior polymorphous endothelial dystrophy, IOL: Intraocular lens

\section{Table 1. Risk factors for Post-Cataract Surgery Corneal} Oedema10

\section{Preoperative Risk Factors}

Pre-Existent Corneal Endothelial Dystrophies

In the backdrop of an endothelial dystrophy, the mechanical stress of a complicated surgery can accelerate the cell loss from the endothelial cell layer that may result in late-onset corneal oedema following surgery.

\section{Iridocorneal Endothelial (ICE) Syndrome}

Chandler syndrome is often associated with early corneal decompensation with a hammered silver appearance on slit lamp examination. This endothelial abnormality may often develop postcataract surgery corneal oedema. ${ }^{2}$

\section{Glaucoma}

Gagnon et al. observed that the endothelial cell counts were inversely proportional to the intraocular pressure and eyes receiving three or four glaucoma medications had lower cell counts than those receiving one or two medications. ${ }^{3}$ The magnitude of the cell loss is usually found to correlate with the duration of rise in IOP and combination with corneal guttata leads to corneal decompensation following cataract extraction. ${ }^{4}$ An eye with shallow anterior chamber (AC) is more likely to encounter multiple intraoperative complications in terms of space for manipulation and recurrent corneolenticular touch. 


\section{Uveitis}

Chronic antecedent anterior segment inflammation is associated with decreased central endothelial cell density, which correlates with the duration of active uveitis, high intraocular pressure during disease and high laser flare photometry value. 5 Moreover, a further insult in the form of surgery may ignite further attacks terminating in acute decompensation of the cornea.

\section{Pseudoexfoliation Syndrome (PXF)}

PXF may be associated with large clumps of typical pseudoexfoliation material, which may be adhered to the corneal endothelium so that the endothelial layer appears irregular and discontinuous. These pathological changes, along with the inherent complications that may possibly occur during the surgery may potentiate acute decompensation in the early as well as late postoperative period. ${ }^{6}$

\section{Trauma}

Endothelial cells around the traumatic lesion undergo the greatest torsion and energy absorption as the cornea moves axially posterior and then relaxes to its original position. ${ }^{7}$ The injury may also be due to a direct contact between the corneal endothelium and a lens or iris. Dysfunction of damaged endothelial cells is replaced with circumferential normal endothelial cells.

\section{Intraoperative Risk Factors}

The various intraoperative factors such as surgeon's experience, instrument trauma, irrigating solutions, duration of surgery, and complications such as vitreous loss can lead to corneal oedema. phacoemulsification leads to corneal endothelial cells damage by generation of free radicals during surgery. Endothelial cells are attached to each other by discontinuous tight junctions that are mainly calcium-dependent. So, the use of calcium-free irrigating solutions during surgery can reduce the barrier function leading to corneal oedema. ${ }^{8}$

\section{Postoperative Risk Factors}

Acute corneal oedema immediately after surgery can be because of endothelial damage by ultrasound energy, inadvertent DM stripping that is DMD, due to the infusion of toxic substances into the $\mathrm{AC}$ that is the toxic anterior segment syndrome (TASS) and IOL endothelial touch. ${ }^{9}$

IOL-related factors, which can often cause corneal decompensation in the postoperative period, include IOL decentration and instability with endothelial touch, dislocated or retained IOL fragments, posterior chamber intraocular lens in $\mathrm{AC}$ and unstable anterior chamber intraocular lens. ${ }^{10}$

\section{Biochemical Alterations in Corneal Oedema}

The endothelial tight junctions on the lateral membranes function in a delicate balance of the biochemical microenvironment. Both calcium and adenosine are required for the barrier function of the endothelium. Paucity of calcium ion or antioxidant glutathione and adenosine contribute to corneal oedema. ${ }^{11}$ The irrigating solutions or drugs used intraoperatively may be a source of insult to the pump functioning of the endothelium. The differential distribution of molecular channels regulating water influx and efflux called aquaporins (AQP) are implicated in affecting the water transport mechanisms within the cornea. AQP abnormalities have been found in Pseudophakic corneal oedema (PCE) corneas (decreased AQP1 and increased AQP3 and AQP4) and Fuchs endothelial corneal dystrophy corneas (decreased AQP). 10

The altered level of integrins leads to upregulation of inflammatory chemical mediators such as insulin-like growth factor 1, transforming growth factor-beta, bone morphogenetic protein 4 (BMP-4), interleukin-1 leading to progressive loss of stromal keratocytes, and formation of a posterior collagenous layer. In these long-standing cases, the corneal epithelium accumulates anti-adhesive proteins with simultaneous loss of adhesive proteins leading to the formation of fluid-filled bullae. ${ }^{10}$

\section{CLINICAL FEATURES Symptoms}

The patients usually present with diminution of vision in the immediate postoperative period with a lack of expected gain in quality of vision. This may be associated with raised IOP and hence symptoms pertaining to the same may be present.

In addition, pain, photophobia, watering, congestion may be present to variable degree due to corneal oedema and associated inflammation. A long-standing corneal oedema may be associated in the initial stages with bullae and the rupture of the same may lead to severe photophobia and pain.

\section{Clinical Examination}

The presence of corneal oedema makes the cornea lustreless and hazy and there may be the concurrent presence of increased corneal thickness on slit lamp biomicroscopy.

On careful evaluation, if the haziness does not preclude it otherwise, a DMD may very well be manifested. A complicated surgery that yielded no particular gain in vision will again be distinguished by the presence of a posterior capsular defect or a poorly stable IOL, with possible vitreous in the $\mathrm{AC}$ and possibly multiple sutures.

As the disease reaches chronicity, in addition to the overlap with aforementioned features, can reveal evidence of scarring, microcysts, severe stromal oedema with or without scarring and bullae [Figs. 1A-C].

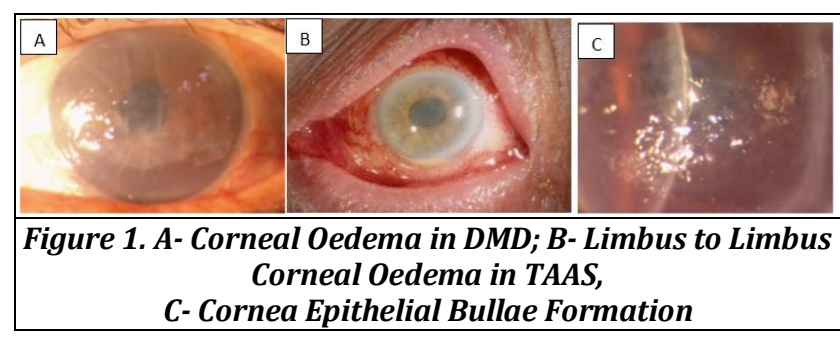

\section{DIFFERENTIAL DIAGNOSIS}

- Toxic Anterior Segment Syndrome: TASS is associated with endothelial failure which heralds diffuse limbus-to-limbus corneal oedema, fibrinous AC reaction, iris atrophy, and trabecular meshwork damage.

- Endophthalmitis: corneal oedema is often associated with vitreous exudates, loss of red glow, ciliary congestion, and severe $\mathrm{AC}$ reaction. 
- Previous Episodes of Angle Closure Attacks: It may reveal iris and pupillary ruff atrophy with gonio synechiae and a pigmented trabecular meshwork.

- Herpetic Endotheliitis: It is associated with KP's, loss of corneal sensation, localized corneal oedema, presence of herpetic footprints/nebulomacular corneal opacity, patchy iris atrophy, and history of recurrent attacks in the past must be looked for.

- Pseudoexfoliation: PXF is distinguished by collection of exfoliated material in the angles with iris atrophy and a poorly dilating pupil that may be detected in the fellow eye.

- Endothelial Dystrophies: multiple central as well as peripheral guttae in the other eye aiding diagnosis. 10

\section{INVESTIGATIONS}

\section{Pachymetry}

Optical or ultrasonic measurements of corneal pachymetry estimate the severity of the corneal oedema. The corneal thickness can be measured with in vivo imaging using OCT, which shows excellent correlation to values obtained by ultrasound pachymetry. 12

\section{Specular Microscopy}

Morphometrical analysis can be done preoperatively in suspect patients such as endothelial dystrophies or with history of multiple precedent surgeries.

\section{Anterior Segment Optical Coherence Tomography (ASOCT)}

In eyes with extensive oedema that precludes clinical examination in detail, the areas and size of DMDs, corneal thickness and levels of scarring can be determined. ${ }^{13}$

\section{Confocal Microscopy}

Confocal microscopy is useful in detecting corneal endothelial status in the presence of corneal oedema. It provides high-quality layer-by-layer analysis of the oedematous cornea thereby providing a clue towards the probable diagnosis.

\section{Management/Medical Management}

Hypertonic Agents (Sodium Chloride 5\% Eye Drops or 6\% Ointment)

These drugs create a hypertonic tear film that draws water out of the oedematous cornea. Hypertonic saline can lead to resolution of corneal oedema in almost one-third of patients, especially in early cases, but the treatment may have to be continued for 3 months. ${ }^{14}$ Hypertonic saline is useful for symptomatic improvement in all cases of corneal oedema. It must be remembered that it does not have any effect on the cause of the disease that must be identified and treated. 10

\section{Bandaged Contact Lenses (BCL)}

Extended-wear hydrophilic contact lenses are useful in reducing pain associated with epithelial bullae. It must be remembered that it does not lead to any reduction in epithelial or stromal oedema. They act by creating an effective precorneal protective layer that shields the swollen epithelium from the lid movement and prevents the rupture of bullae. 14
Hydrophilic extended-wear contact lenses along with hypertonic saline drops can be used to create a hypertonic reservoir. This reservoir continuously bathes the cornea, providing corneal deturgescence for a relatively longer period.

\section{Antiglaucoma Drugs}

Raised IOP can be managed with topical antiglaucoma medications or surgical options such as trabeculectomy with mitomycin $\mathrm{C}$ or a glaucoma drainage implant in cases not controlled with topical therapy. Lowering the IOP not only improves the corneal oedema but also prevents further damage to endothelium.

\section{Topical Steroids}

Associated inflammation must be treated with topical steroids. It is better to avoid using steroids at a higher frequency since associated problems like raise in IOP may further delay clearing of corneal oedema.

Management of TASS, in the acute phase, includes the intensive use of topical corticosteroids and systemic steroids (In severe cases) along with the use of antiglaucoma drugs. In late phase, development of endothelial decompensation requires keratoplasty.

\section{Surgical Management}

Spontaneous reattachment is commonly seen in planar and nonscrolled DMD's within days after surgery. ${ }^{14}$ It has been reported by Mackool and Holtz that intervention is often required in cases of extensive, central, nonplanar DMD with scrolled or torn edges. 15

\section{Descemetopexy}

Sparks first described this procedure in three eyes with extensive DMDs. ${ }^{16}$ Three major tamponade agents used are air, $15 \%-20 \%$ SF6 and $12 \%-14 \%$ C3F8. Due to the fast absorption of air, SF6 and C3F8 are the preferred agents [Fig. 2].

The major complication reported is pupillary block (7.7\%), which can be prevented and managed with the use of cycloplegics, prophylactic laser iridotomy, oral and topical antiglaucoma drugs, or a partial fill of AC with air or gas.

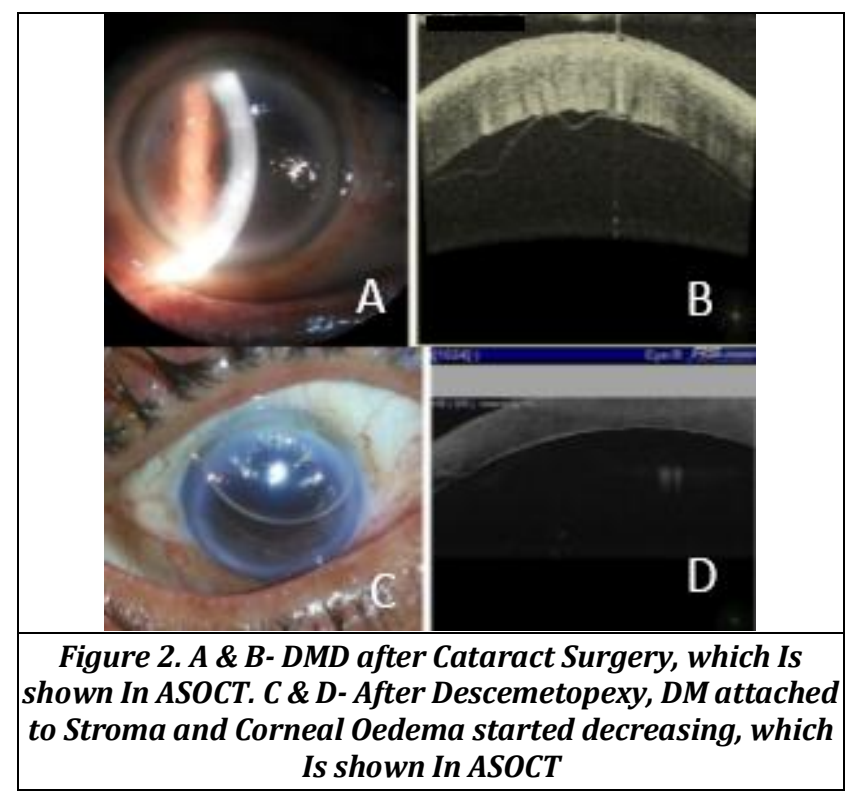


Thus, the standard treatment option remains descemetopexy. However, other surgical options are viscoelastic injection, suture fixation, and endothelial or penetrating keratoplasty in cases where DMD progress to a stage of corneal decompensation. 10

The time interval between cataract surgery and Descemet stripping endothelial keratoplasty (DSEK) is a critical factor in determining long-term success and a minimum 36-month waiting period after cataract is essential for optimal outcomes. Surgery, if performed within 3 months was seen to be associated with dismal visual outcome and often a repeat graft is required. ${ }^{17}$

\section{CONCLUSION}

Corneal oedema following cataract surgery is an untoward but avoidable complication in most of the cases. A careful preoperative workup, intraoperative precautions and vigilant postoperative care can avoid this complication. Even though, if it happens, then first cause of corneal oedema should be explored by slit-lamp examination and other investigative modalities. If TAAS is there, it should be managed medically. If Descemet membrane detachment is present, then descemetopexy with air or gas should be considered sooner than later. Endophthalmitis is a vision threatening cause, hence it should be treated urgently. Other causes of corneal oedema should be tackled accordingly. Even after all the efforts if corneal oedema does not subside and corneal decompensation happened, then endothelial keratoplasty can be done after 3 months of cataract surgery.

\section{REFERENCES}

[1] Maurice DM. The structure and transparency of the cornea. J Physiol 1957;136(2):263-86.

[2] Yi DH, Dana MR. Corneal oedema after cataract surgery: incidence and etiology. Semin Ophthalmol 2002;17(3-4):110-4.

[3] Gagnon MM, Boisjoly HM, Brunette I, et al. Corneal endothelial cell density in glaucoma. Cornea 1997;16(3):314-8.

[4] Bigar F, Witmer R. Corneal endothelial changes in primary acute angle-closure glaucoma. Ophthalmology 1982;89(6):596-9.

[5] Alfawaz AM, Holland GN, Yu F, et al. Corneal endothelium in patients with anterior uveitis. Ophthalmology 2016;123(8):1637-45.
[6] Schlötzer-Schrehardt UM, Dörfler S, Naumann GO. Corneal endothelial involvement in pseudoexfoliation syndrome. Arch Ophthalmol 1993;111(5):666-74.

[7] Maloney WF, Colvard M, Bourne WM, et al. Specular microscopy of traumatic posterior annular keratopathy. Arch Ophthalmol 1979;97(9):1647-50.

[8] Rubowitz A, Assia EI, Rosner M, et al. Antioxidant protection against corneal damage by free radicals during phacoemulsification. Invest Ophthalmol Vis Sci 2003;44(5):1866-70.

[9] Benatti CA, Tsao JZ, Afshari NA. Descemet membrane detachment during cataract surgery: etiology and management. Curr Opin Ophthalmol 2017;28(1):35-41.

[10] Sharma N, Singhal D, Nair SP, et al. Corneal oedema after phacoemulsification. Indian J Ophthalmol 2017;65(12):1381-9.

[11] Riley MV, Winkler BS, Starnes CA, et al. Regulation of corneal endothelial barrier function by adenosine, cyclic AMP, and protein kinases. Invest Ophthalmol Vis Sci 1998;39(11):2076-84.

[12] Bechmann M, Thiel MJ, Neubauer AS, et al. Central corneal thickness measurement with a retinal optical coherence tomography device versus standard ultrasonic pachymetry. Cornea 2001;20(1):50-4.

[13] Sharma N, Gupta S, Maharana P, et al. Anterior segment optical coherence tomography-guided management algorithm for Descemet membrane detachment after intraocular surgery. Cornea 2015;34(9):1170-4.

[14] Narayanan R, Gaster RN, Kenney MC. Pseudophakic corneal oedema: a review of mechanisms and treatments. Cornea 2006;25(9):993-1004.

[15] Mackool RJ, Holtz SJ. Descemet membrane detachment. Arch Ophthalmol 1977;95(3):459-63.

[16] Sparks GM. Descemetopexy. Surgical reattachment of stripped Descemet's membrane. Arch Ophthalmol 1967;78(1):31-4.

[17] Kaur M, Titiyal JS, Falera R, et al. Outcomes of descemet stripping automated endothelial keratoplasty in toxic anterior segment syndrome after phacoemulsification. Cornea 2017;36(1):17-20. 